\title{
Effectiveness of a standard protocol for tranexamic acid administration in total hip and knee arthroplasty - does it make a difference?
}

\author{
Sudhan S R. ${ }^{1 *}$, Karthick S. ${ }^{2}$, Nagendra V. ${ }^{3}$, Asokan J. ${ }^{4}$, George V. ${ }^{5}$, Jacob J. ${ }^{6}$ \\ DOI: https://doi.org/10.17511/ijoso.2020.i02.09 \\ 1* Ram Sudhan S, Junior Consultant, Department of Orthopaedics, Sunrise Hospital, Changaramukulam, Kerala, India. \\ https://orcid.org/0000-0002-6327-3861 \\ 2 Srinivas Karthick, Junior Consultant, Department of Orthopaedics, Medical Trust Hospital, Ernakulam, Kerala, India. \\ 3 Vijetha Nagendra, Senior Registrar, Department of Neuroanaesthesia and Neurocritical care, Apollo Hospitals, Bangalore, Karnataka, \\ India. \\ ${ }^{4}$ Jithesh Asokan, Consultant, Department of Anaesthesia, Medical Trust Hospital, Ernakulam, Kerala, India. \\ 5 Vinod George, Associate Consultant, Department of Orthopaedics, Medical Trust Hospital, Ernakulam, Kerala, India. \\ 6 Jacob J, Consultant, Department of Orthopaedics, Medical Trust Hospital, Ernakulam, Kerala, India.
}

Background: Intraoperative blood loss plays a key role in the outcome of any surgery and is most important during the immediate post-operative period in the management, for both the operating surgeon and the anesthetist. Methods: The primary aim of the study was to prove the efficiency of standardized tranexamic acid administration in reducing blood loss and need for blood transfusion in total knee and hip arthroplasty by comparing postoperative hemoglobin levels, peri-operative hemoglobin drop and frequency of blood transfusion in 2 groups one receiving and the other not receiving tranexamic acid during the peri-operative period. Results: The mean pre-operative hemoglobin in the tranexamic acid group was 13.45 and in the control group was 13.0 with a pvalue of 0.267 , The mean hemoglobin level as on a postoperative day 1 in the tranexamic acid group was 12.32 and in the control group was 10.78 with p-value $<0.001$. Conclusion: The use of standardized Tranexamic acid infusion during the perioperative period of Total Hip and Knee Arthroplasty reduced the perioperative blood loss which was evident by high mean postoperative hemoglobin levels and low mean postoperative hemoglobin drop and decreased need for blood transfusion in Tranexamic acid group when compared to the control group.

Keywords: Total Knee Arthroplasty, Total Hip Arthroplasty, Tranexamic Acid

\section{Corresponding Author}

Ram Sudhan S, Junior Consultant, Department of Orthopaedics, Sunrise Hospital, Changaramukulam, Kerala, India.

Email: sudhansubramaniam@gmail.com

\section{How to Cite this Article}

Sudhan SR, Karthick S, Nagendra V, Asokan J, George V, Jacob J. Effectiveness of a standard protocol for tranexamic acid administration in total hip and knee arthroplasty - does it make a difference?. Surgical Review Int J Surg Trauma Orthoped. 2020;6(2):121-126.

Available From: https://surgical.medresearch.in/ index.php/ijoso/article/view/170

\section{To Browse}

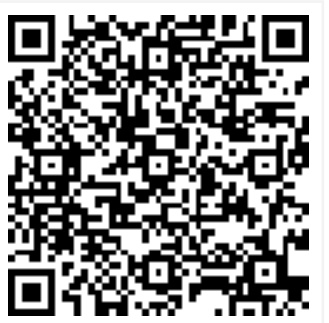

Manuscript Received 06-04-2020

Conflict of Interest No
Review Round 1 16-04-2020

Funding Nil

Review Round 2
22-04-2020
Ethical Approval
yes

Review Round 3

Plagiarism X-checker $14 \%$
Accepted 28-04-2020

(C) 2020 by Ram Sudhan S, Srinivas Karthick, Vijetha Nagendra, Jithesh Asokan, Vinod George, Jacob J and Published by Siddharth Health Research and Social Welfare Society. This is an Open Access article licensed under a Creative Commons Attribution 4.0 International License https://creativecommons.org/licenses/by/4.0/ unported [CC BY 4.0]. 


\section{Introduction}

The number of Total Knee Arthroplasty (TKA) and Total Hip Arthroplasty (THA) has been markedly increasing in the last few decades due to an increase in life expectancy [1]. The evolving nature of mankind in pursuing improved quality of life demands precision in the protocol for arthroplasty, and thus it emerged as the most performed surgery in the western world and India [1].

Total hip replacement has been widely acclaimed as the "Operation of the century" and Substantial blood loss in perioperative and postoperative periods is inevitable and it affects the outcome of surgery and may result in increased morbidity, mortality, and length of hospital stay [2]. Thus, it becomes mandatory to control the blood loss to improve the outcome of surgery and to decrease the disability days and the economic burden of patients. Several methods of conservation of blood have been used that include autologous blood transfusion, Intraoperative blood salvage, medical management by use of erythropoietin, and antifibrinolytic agents $[3,4]$.

Blood transfusion can be accompanied by rare, lifethreatening complications, including acute and delayed hemolytic reactions, acute lung injury, and disease transmission [5]. Several observational studies have raised concerns over the association of blood transfusion with poorer outcomes after surgery, such as including longer length of hospital stay, economic concerns, increased infections, and mortality [6]. Various studies have assessed the effectiveness of antifibrinolytics (Tranexamic acid TXA) in patients of Total Knee Arthroplasty and Total Hip Arthroplasty which showed promising results [7].

Anti-Fibrinolytic action of TXA and aprotinin has been proven to reduce blood loss in surgeries [8] and TXA is cheaper and more specific fibrinolytic inhibitor than aprotinin and has been shown to reduce blood loss in arthroplasties $[9,10]$. The efficacies of TXA in TKA and THA remains uncertain due to varied protocol of administration (time and dosage of administration of TXA) and have yielded contrasting results varying from decreased blood loss, decreased blood transfusion requirements to no difference in perioperative blood loss and even increased transfusion requirements [11-13].

This prompted us to evaluate the efficacy of TXA with a standardized protocol of administration.

\section{Material and Methods}

This prospective randomized study was conducted in the Department of Orthopaedics, Medical Trust Hospital, Ernakulam, Kerala from December 2016 to Dec.2017. Institutional Ethical committee permission was obtained for the Study. Written consent was obtained for the study.

\section{Inclusion criteria}

01. Patients age between 30-80 years who are undergoing THA and

02. Patients with weight $50-100$

03. Patients with the ability to provide informed consent and to co-operate with the

\section{Exclusion criteria}

01. Patients with major co-morbidities.

02. Patients with renal

03. Patients with abnormal liver

04. Patients with a major neurological

The study interventions were perioperative standard $1 \mathrm{gm}$ intravenous Tranexamic acid infusion with $100 \mathrm{ml}$ NS (Tranexamic Acid Group/TXA group) versus No-tranexamic acid [Control group].

Patients will be included, evaluated, and studied after explaining the procedures in detail and after getting the patient's consent. A total of $n=41$ patients have been included in the study and were studied.

Patients are randomized using the numbers generated in the range from 0 to 99 by a computer software program (Microsoft Excel), each time when a patient is enrolled in the trial, a generated random number is allotted, such that patients with even numbers were taken for Tranexamic acid group (TXA Group) and odd numbers were taken for Control group.

Table 1: Blood transfusion protocol.

\begin{tabular}{|l|l|}
\hline \multicolumn{1}{|c|}{$\begin{array}{c}\text { Hemoglobin } \\
\text { levels }\end{array}$} & \multicolumn{1}{|c|}{ Blood transfusion } \\
\hline$<8 \mathrm{mg} / \mathrm{dl}$ & $\begin{array}{l}\text { Blood transfusion irrespective of signs and symptoms of } \\
\text { low volume }\end{array}$ \\
\hline $8 \mathrm{mg} / \mathrm{dl}-10$ & Blood transfusion only if patients were symptomatic \\
$\mathrm{mg} / \mathrm{dl}$ & \\
\hline$>10 \mathrm{mg} / \mathrm{dl}$ & No transfusion \\
\hline
\end{tabular}

All patients undergoing TKA and THA were receiving a standard perioperative dose of $1 \mathrm{gm}$ of Tranexamic acid given intravenously with $100 \mathrm{ml}$ Normal saline 
Immediately after spinal anesthesia, preoperative hemoglobin levels (at 12 hours prior to surgery) and postoperative hemoglobin levels are recorded (at 6 hours postoperatively, 24 hours and 72 hours thereafter) and compared with patients who have not received tranexamic acid, frequency of blood transfusion in both the groups were noted and compared. Standard institutional blood transfusion protocols were followed in all patients regardless of the diagnosis (Primary OA, RA, Post Traumatic OA), TKA is done by medial Parapatellar approach, a tourniquet was used for all patients, no drain was used in THA irrespective of diagnosis and was done through standard lateral approach in the lateral position without a drain, other perioperative interventions such as spinal anesthesia, prophylactic antibiotics, knee prostheses, and hip prostheses and thromboprophylaxis were kept similar in all patients. Post-operatively all patients will undergo the same standardized rehabilitation protocol.

\section{Parameters observed}

01. Preoperative hemoglobin levels of all the patients of Tranexamic acid group and control group (at 12 hours prior to surgery),

02. Postoperative hemoglobin levels of both the groups (at 6 hours postoperatively, 24 hours and 72 hours thereafter),

03. Frequency of blood transfusions in both the groups,

04. Complications if any in patients who were given Tranexamic acid and those who are undergoing blood transfusion (observed for a period of 30 days).

Patients did not receive any premedication. In both treatment groups, paracetamol $1 \mathrm{~g}$ iv infusion 8 hourly and after 48 hours converted to Oral tablet Aceclofenac $100 \mathrm{mg} 12$ hourly and capsule Omeprazole $20 \mathrm{mg}$ once a day. Opioid (Tramadol Hydrochloride $50 \mathrm{mg}$ ) with centrally acting antiemetic Ondansetron $4 \mathrm{mg}$ SOS is used as rescue analgesia. 2nd generation cephalosporin and aminoglycoside will be used as prophylactic antibiotics as intravenous (IV) perioperatively and for every 12 hours for the first 48 hours of surgery. For thromboprophylaxis, Injection Dalteparin 2500 IU subcutaneously once each evening (at $6 \mathrm{pm}$ ) will be given till the day of discharge from the postoperative day (POD)-0. Bilateral flowtron pumps were also used for all the patients. Range of movement exercises was started from day 1 ,
Patients are made to stand and walk on flat ground on POD 1 and climb steps on POD 2. Suture removal was done on the 14th postoperative day. Patients were followed on an outpatient basis after 1 month followed by every 2 months for one year. The technique of giving Tranexamic Acid: All the patients who included in the tranexamic acid group received a standard dose of $1 \mathrm{gm}$ of tranexamic acid diluted in $100 \mathrm{ml}$ of normal saline and given over 10 minutes, immediately after spinal anesthesia. Two vials of tranexamic acid each $500 \mathrm{mg}$ were used. The statistical analysis will be performed by IBM SPSS Statistics 20 version Categorical variables will be described as frequency and percentage.

\section{Results}

A total of $n=41$ patients underwent THA and TKA during the study period and were eligible for inclusion in the study. $\mathrm{N}=20$ patients in the group were given Tranexamic Acid prior to the commencement of surgery after spinal anesthesia, and $n=21$ patients were not given Tranexamic acid. The mean age of patients in Tranexamic Acid Group [TXA group] is $60.40 \pm 12$ and the Control Group is $60.23 \pm 12.31$. The difference between the two groups was not statistically significant $(P=0.967)$. The sex distribution among TXA Group and Control Group was comparable and there was no significant difference 0.09 .

Table 2: Comparison of age between the two groups of the study.

\begin{tabular}{|l|l|l|}
\hline \multicolumn{1}{|c|}{ Study Group } & \multicolumn{1}{c|}{ Mean \pm S.D } & \multicolumn{1}{c|}{ P-Value } \\
\hline Control Group & $60.23 \pm 12.31$ & 0.967 \\
\cline { 1 - 2 } TXA Group & $60.40 \pm 12.84$ & \\
\hline
\end{tabular}

Table 3: Comparison of sex between the TXA group and the control group.

\begin{tabular}{|l|l|l|l|}
\hline \multirow{2}{*}{ Study Group } & \multicolumn{2}{c|}{ Sex } & \multirow{2}{*}{ Total } \\
\cline { 2 - 3 } & Female & Male & \\
\hline Control Group & 13 & 8 & 21 \\
\hline TXA Group & 17 & 3 & 20 \\
\hline Total & 30 & 11 & 41 \\
\hline
\end{tabular}

P-value $=0.09$

Table-4: Distribution of surgical procedure in the study group.

\begin{tabular}{|l|l|l|l|l|l|}
\hline \multirow{3}{*}{ Study Group } & LEFT & LEFT & \multicolumn{1}{|c|}{ RIGHT } & \multicolumn{1}{|c|}{ RIGHT } & \multirow{2}{*}{ TOTAL } \\
\cline { 2 - 5 } & THR & TKR & THR & TKR & \\
\hline Control group & 3 & 4 & 5 & 9 & 21 \\
\hline TXA group & 5 & 3 & 3 & 9 & 20 \\
\hline Total & 8 & 7 & 8 & 18 & 41 \\
\hline
\end{tabular}


In this prospective randomized controlled study, evaluation, and comparison of the efficiency of 'single, standard Intravenous dose of Tranexamic acid' in the group of 41 patients who underwent THA and TKA was done. There was no significant difference in the demographic profile including age, sex, and diagnosis of the subjects compared, ensuring comparability and matching between the two groups i.e. Tranexaimic Acid Group and Control Group. Postoperative hemoglobin levels on a postoperative day $[\mathrm{POD}] 1$ and postoperative day $[P O D] 3$ were higher in subjects undergoing THA and TKA who were receiving Standard Intravenous dose of $1 \mathrm{gm}$ tranexamic acid than subject undergoing THA and TKA and not receiving tranexamic acid when compared individually, and their Mean implied the same results.

The hemoglobin decrease from preoperative level to postoperative day 1 and postoperative day 3 was less in subjects undergoing THA and TKA and receiving Standard intravenous dose of $1 \mathrm{gm}$ tranexamic acid than subjects undergoing THA and TKA and not receiving tranexamic acid when compared individually and their Mean implied the same results.

Table-5: Mean hemoglobin level of both the groups at POD 1 and POD 3.

\begin{tabular}{|c|c|c|}
\hline Mean \pm S.D & & P-Value \\
\hline \multicolumn{3}{|c|}{ Postoperative Day 1} \\
\hline Control group & $10.78 \pm 1.36$ & \multirow[t]{2}{*}{$<0.001$} \\
\hline TXA group & $12.32 \pm 1.34$ & \\
\hline \multicolumn{3}{|c|}{ Postoperative Day 3} \\
\hline Control group & $9.31 \pm 1.40$ & \multirow[t]{2}{*}{$<0.001$} \\
\hline TXA group & $11.75 \pm 1.35$ & \\
\hline
\end{tabular}

Table-6: Requirements for blood transfusion in both groups.

\begin{tabular}{|l|l|l|l|}
\hline \multirow{2}{*}{ Study Group } & \multicolumn{2}{c|}{ Blood Transfusion } & \multirow{2}{*}{ Total } \\
\cline { 2 - 3 } & No & Yes & \\
\hline Control group & 18 & 3 & 21 \\
\hline TXA group & 20 & 0 & 20 \\
\hline Total & 38 & 3 & 41 \\
\hline
\end{tabular}

\section{Discussion}

The need for blood transfusion during the postoperative period in the group receiving tranexamic acid during the perioperative period was nil when compared with the control group not receiving perioperative tranexamic acid. The findings of the present study were in agreement with Hiippala S et al; [14] in their prospective,
Randomized, double-blind study, had investigated the effect of Tranexamic acid on blood loss and transfusion requirements associated with total knee arthroplasty group and concluded that tranexamic acid reduced perioperative blood loss and transfusion requirements associated with TKA.

Johansson T et al; [15] investigated the effects of a single bolus of $15 \mathrm{mg} / \mathrm{kg}$ IV TXA given at the start of the primary THA and found a significant reduction in both blood loss and the need for postoperative transfusion. Alvarez JC et al; [16] in a prospective, randomized, double-blinded study, assessed the effectiveness of IV TXA in patients undergoing primary TKA and receiving $10 \mathrm{mg} / \mathrm{kg}$ of IV TXA before tourniquet release, supplemented by a 1 $\mathrm{mg} / \mathrm{kg} / \mathrm{h}$ infusion for 6 hours postoperatively.

The TXA-administered group showed a significant reduction in blood loss and transfusion requirements. There were no significant differences in venous thromboembolic events in the TXA group compared with the control group. Similarly with Zufferey PJ et al; [17] conducted on a variety of hip fracture types, shows a decreased number of transfusions in the TXA group, however, this did not have statistical significance. Similarly with Alshryda $S$ et al; [18] compared topical tranexamic acid versus normal saline administration in $n=161$ patients undergoing unilateral primary THA.

A total of $n=81$ patients received normal saline, and $\mathrm{n}=80$ patients received $1 \mathrm{~g}$ of topical TXA. The TXA group had a higher post-operative hemoglobin level and a significant decrease in the transfusion rate from $32.1 \%$ to $12.5 \%$ in the group receiving $1 \mathrm{~g}$ of topical TA, though his study used topical tranexamic acid rather than Intravenous Tranexamic acid, the efficiency of tranexamic acid in reducing blood loss and hemoglobin decrease and Blood transfusion requirements can be confirmed.

In the present study, patients treated with TXA never required transfusions, and there were no differences in symptomatic thromboembolic events within 30 days of surgery between patients who received TXA and those who did not. And especially in high-risk patients, TXA was not associated with an increase in symptomatic thromboembolic events. Thus, it facilitated them to mobilize early and to return to normal daily activities faster, thereby reducing the duration of hospital stay and days of disability. Similar results were observed in blood loss and venous thromboembolic events by Wei $Z$ et al; [19] who conducted a meta-analysis that 
Combined all data from available randomized controlled trials, regardless of the methods of TXA administration, which included intravenous, intraarticular, topical or oral. CD Watts et al; [20] (2017) in their study 138 patients presented with a lowenergy, isolated, Fracture neck of femur treated with either hemi or total hip arthroplasty. In the TXA group, patients received 2 doses of $15 \mathrm{mg} / \mathrm{kg}$ intravenous TXA dissolved in $100 \mathrm{ml}$ of saline; each administered over 10 minutes; 1 dose just before incision, and the second at wound closure.

In the placebo group, $100 \mathrm{ml}$ of saline solution was administered similarly. There was a trend toward decreased transfusion rate in the TXA group (17\% vs. $26 \%, P=0.22)$. TXA was safe with no differences in adverse events at 30 and 90 days and concluded that the TXA administration safely reduces blood loss with a tendency for decreased transfusion rate and total blood product consumption for patients undergoing hip arthroplasty for acute fracture neck of femur. More studies are needed to further ascertain the role of TXA in the management of patients with Fracture neck of femur.

\section{Conclusion}

The use of a standard protocol and dose of intravenous tranexamic acid during the perioperative period of THA and TKA reduced the perioperative blood loss which was evident by higher mean postoperative hemoglobin levels and low mean hemoglobin drop thus decreasing the need for blood transfusion in the Tranexamic acid group when compared to the control group.

\section{What does the study add to the existing knowledge?}

Thus, the administration of Tranexamic acid greatly reduces the complications, morbidity, and duration of hospital stay and decreases the disability period thereby improving the standard of arthroplasty and quality of life.

\section{Author's contribution}

All the authors, Dr. Ram Sudhan S., Dr. Srinivas Karthick, Dr. Vijetha Nagendra, Dr. Jithesh Asokan, Dr. Vinod George, Dr. Jacob J. jointly collaborated during the study design, conduct of the study, statistical analysis and during the manuscript preparation.

\section{Reference}

01. Paul JE, Arya A, Hurlburt L, Cheng J, Thabane L, Tidy $A$, et al. Femoral Nerve Block Improves Analgesia Outcomes after Total Knee ArthroplastyA Meta-analysis of Randomized Controlled Trials. Anesthesiology. 2010;113(5)1144-1162.

doi: [Article:https://doi.org/10.1097/aln.0b013e31 81f4b18][Crossref]

02. Carson JL, Duff A, Poses RM, Berlin JA, Spence RK, Trout $R$, et al. Effect of anemia and cardiovascular disease on surgical mortality and morbidity. Lancet. 1996;348(9034)1055-1060.

[Crossref]

03. Cardone D, Klein AA. Perioperative blood conservation. Eur J Anaesthesiol. 2009;26(9)722-729.

doi: [Article:https://doi.org/10.1097/eja.0b013e32 832c5280][Crossref]

04. Rajesparan K, Biant LC, Ahmad M, Field RE. The effect of an intravenous bolus of tranexamic acid on blood loss in total hip replacement. J Bone Joint Surg Br. 2009;91(6)776-783. doi:[Article:https://doi.org/10.1302/0301-620x. 91b6.22393][Crossref]

05. Bolton Maggs PH, Cohen H. Serious Hazards of Transfusion (SHOT) haemovigilance and progress are improving transfusion safety. $\mathrm{Br} \mathrm{J}$ Hematol. 2013;163(3)303-314.

doi:[Article:https://doi.org/10.1111/bjh.12547] [Crossref]

06. Frisch NB, Wessell NM, Charters MA, Yu S, Jeffries JJ, Silverton CD. Predictors and complications of blood transfusion in total hip and knee arthroplasty. J Arthroplasty. 2014;29(9)189-192.

doi:[Article:https://doi.org/10.1016/j.arth.2014.03. 048][Crossref]

07. Hallstrom B, Singal B, Cowen ME, Roberts KC, Hughes RE. The Michigan experience with safety and effectiveness of tranexamic acid use in hip and knee arthroplasty. J Bone Joint Surg. 2016;98(19)1646-1645.

[Crossref]

08. Shore-Lesserson L, Reich DL, Vela-Cantos $F$, Ammar T, Ergin MA. Tranexamic acid reduces transfusions and mediastinal drainage in repeat cardiac surgery. Anesth Analg. 1996;83(1)1826.

[Crossref] 
09. Benoni G, Fredin HA. Fibrinolytic inhibition with tranexamic acid reduces blood loss and blood transfusion after knee arthroplasty. Bone Joint J. $1996 ; 78(3) 434-440$.

[Crossref]

10. Watts CD, Houdek MT, Sems SA, Cross WW, Pagnano MW. Tranexamic Acid Safely Reduced Blood Loss in Hemi-and Total Hip Arthroplasty for Acute Femoral Neck Fracture- A Randomized Clinical Trial. J Orthop Trauma. 2017;31(7)345351.

doi:[Article:https://doi.org/10.1097/bot.00000000 00000837][Crossref]

11. Molloy DO, Archbold HA, Ogonda L, McConway J, Wilson RK, Beverland DE. Comparison of topical fibrin spray and tranexamic acid on blood loss after total knee replacement. Bone Joint J. 2007;89(3)306-309.

[Crossref]

12. Ekbäck G, Axelsson K, Ryttberg L, Edlund B, Kjellberg J, Weckström J, et al. Tranexamic acid reduces blood loss in total hip replacement surgery. Anesth Analg. 2000;91(5)1124-1130. [Crossref]

13. Niskanen RO, Korkala OL. Tranexamic acid reduces blood loss in cemented hip arthroplastya randomized, double-blind study of 39 patients with osteoarthritis. Acta Orthopaedica. $2005 ; 76(6) 829-832$.

[Crossref]

14. Husted H, Blønd L, Sonne-Holm S, Holm G, Jacobsen T, Gebuhr P. Tranexamic acid reduces blood loss and blood transfusions in primary total hip arthroplasty A prospective randomized double-blind study in 40 patients. Acta Orthopaedica Scandinavica. 2003;74(6)665669.

[Crossref]

15. Alvarez JC, Santiveri FX, Ramos I, Vela E, Puig L, Escolano F. Tranexamic acid reduces blood transfusion in total knee arthroplasty even when a blood conservation program is applied. Transfusion. 2008;48(3)519-525. doi:[Article:https://doi.org/10.1111/j.1537-2995. 2007.01564.x][Crossref]

16. Zufferey PJ, Miquet M, Quenet $S$, Martin $P$, Adam $\mathrm{P}$, Albaladejo $\mathrm{P}$, et al. Tranexamic acid in hip fracture surgery- a randomized controlled trial. $\mathrm{Br}$ J Anesth. 2009;104(1)23-30.

doi: [Article:https://doi.org/10.1093/bja/aep314] [Crossref]
17. Hiippala S, Strid L, Wennerstrand M, Arvela V, Mäntylä S, Ylinen J, Niemelä H. Tranexamic acid (Cyklokapron) reduces perioperative blood loss associated with total knee arthroplasty. BJA- $\mathrm{Br}$ J Anaesth. 1995;74(5)534-537.

[Crossref]

18. Wei Z, Liu M. The effectiveness and safety of tranexamic acid in total hip or knee arthroplasty- a metaanalysis of 2720 cases. Transfusion Med. 2015;25(3)151-162.

doi: [Article:https://doi.org/10.1111/tme.12212] [Crossref]

19. Alshryda S, Mason J, Vaghela M, Sarda P, Nargol A, Maheswaran $S$, et al. Topical (intra-articular) tranexamic acid reduces blood loss and transfusion rates following total knee replacement- a randomized controlled trial (TRANX-K). J Bone Joint Surg Am. 2013;95(21)1961-1968.

doi: [Article:https://doi.org/10.2106/jbjs.l.00907] [Crossref]

20. Johansson T, Pettersson LG, Lisander B. Tranexamic acid in total hip arthroplasty saves blood and money- a randomized, double-blind study in 100 patients. Acta Orthop. 2005;76(3)314-319.

[Crossref] 